\title{
Retraction of: \\ A Novel CircRNA Circ_0095424 Regulates Proliferation, Metastasis, and Apoptosis of Osteosarcoma Cells Via the PI3K/AKT Signaling Pathway Through Targeting the miR-1238/HMGB1 Axis by Zhang et al., Cancer Biother Radiopharm epub 19 Aug 2020; DOI: $10.1089 /$ cbr.2020.3563
}

\footnotetext{
$C$ ANCER BIOTHERAPY AND RADIOPHARMACEUTICALS is officially retracting the article entitled, A Novel CircRNA Circ_0095424 Regulates Proliferation, Metastasis, and Apoptosis of Osteosarcoma Cells Via the PI3K/AKT Signaling Pathway Through Targeting the miR-1238/HMGB1 Axis by Zhang et al., (Cancer Biother Radiopharm epub 19 Aug 2020 ; DOI: 10.1089/cbr.2020.3563), due to manipulated images appearing in the published paper.
}

The Editor of the journal received an email on August 31, 2020 from the corresponding author of the article, Dr. Chuan Qin, indicating that, "due to our negligence in organizing the pictures, the protein pictures are repeatedly placed in Figure $7 G$ PI3K. For this, we express our sincerest apologies. We need to [issue] an [erratum] on this issue. We have replaced the protein picture of Figure $7 G$ with the correct picture." However, one of the attachments submitted with the request appeared to be the original version of Figure 7 from the accepted manuscript. A second attachment appeared to be the data from three replicates to be used (by the journal) to construct a revised version of Figure 7 . The Editor, in turn, informed the authors that it was not at the journal's discretion to create a new image for them, and asked the authors to create the revised figure and supply it to the publisher. Below is the response from Dr. Qin, dated September 2, 2020.

“In fact, our team's Western blot experiment commissioned a third-party company for testing. At present, some peers have found that the company has forged experimental reports. We believe that the authenticity of the data provided by the company is problematic. After contacting the company, they were unable to provide the original images. In view of the problems in this manuscript, all the authors discussed and agreed to withdraw the manuscript."

As the entirety of the situation is unacceptable, the Editor officially retracts the article based on the "forged experimental reports" and the questionable validity of the data provided.

The Editor and Publisher of Cancer Biotherapy and Radiopharmaceuticals is dedicated to preserving the integrity of the scientific literature and the community it serves. 